\title{
Numerical and theoretical investigation of the surface flows of granular materials on heaps
}

\author{
A. de Ryck ${ }^{\text {a }}$, H.P. Zhu ${ }^{\text {b,c,*, S.M. Wu }}{ }^{\text {b }}$, A.B. Yu ${ }^{\text {b }}$, P. Zulli ${ }^{d}$ \\ a École des Mines d'Albi-Carmaux, centre Rapsodee, FRE CNRS 3213, route de Teillet, F-81000, Albi, France \\ b Laboratory for Simulation and Modelling of Particulate Systems, School of Materials Science and Engineering, The University of New South Wales, Sydney, NSW 2052, Australia \\ c School of Engineering, University of Western Sydney, Locked Bag 1797, Penrith South DC, NSW 1797, Australia \\ d BlueScope Steel Research Laboratories, P.O. Box 202, Port Kembla, NSW 2505, Australia
}

\begin{abstract}
A B S T R A C T
Flows of granular material on 'non-frozen' and 'frozen' heaps are considered numerically and theoretically in this work. The surface flow on a 'non-frozen' heap is first investigated numerically using the discrete element method. The flow profiles of the surface granular flow and the creep motion of particles in the heap are studied. It is shown that the mean velocity of the surface flow exhibits a linear relationship with the distance from the heap surface, while that of the particles in the heap decays exponentially with the distance. The existence of such a creep motion may be attributed to the variation of the porosity distribution of the heap. The granular flow on a 'frozen' static heap is also simulated, and compared with the one on the corresponding 'non-frozen' heap. The results show that the surface conditions of the heap, to some extent, affect the flow upon it. The surface flow on a static heap is then theoretically examined in detail by using a recently developed continuum model. The depth of the steady-state surface flow and its dependence on wall friction and heap width are investigated. In addition, the theoretical results are compared with the DEM simulation results and the experimental ones in the literature, and qualitatively good agreements are observed.
\end{abstract}

Keywords:

Granular flow

Heap

Discrete element method

Continuum model

\section{Introduction}

Surface flow of granular materials is often observed in many industrial processes such as those in mining, iron-making, chemical and civil engineering [1], and geophysical situations such as landslides [2] and snow avalanches [3]. The study of the dynamical behaviour of such flow is of significance for formulation of optimal design and control strategies under industrial conditions and development of the rheology of granular material $[4,5]$.

In the past, many theoretical, numerical and experimental efforts have been conducted to study surface flows on heaps. Bagnold [6] contributed his pioneered work on the fundamental laws at work inside surface flows. Savage and Hutter [4] developed a general model to describe the flows based on depth-averaged mass and momentum balance equations. Pouliquen and Renaut [7] investigated the effect of the dilatancy on the onset of granular flows on an inclined rough surface. Brewster et al. [8] studied the plug flow and breakdown of Bagnold scaling in cohesive flows. Jop et al. [9] proposed a continuum model for surface flows by introducing a strain rate law in the constant

\footnotetext{
* Corresponding author. School of Engineering, University of Western Sydney, Locked Bag 1797, Penrith South DC, NSW 1797, Australia. Tel.: +61 247360108 ; fax: +61247360833 .

E-mail address: h.zhu@uws.edu.au (H.P. Zhu).
}

friction model developed by Jenike [10]. de Ryck et al. [11] and de Ryck [12] developed the model of Jop et al. [9] to investigate the velocity field of parallel flows of non-cohesive and cohesive particles. These studies are mainly restricted to the flows over static heaps (referred to as 'frozen' heaps here) or chutes. Recently, the experimental study of Komatsu et al. [13] and the numerical study of Richard et al. [14] suggest that in the case of free surface flows on a heap (referred to as 'non-frozen' heap here), even the particles in the deep of the heap still exhibit slow flow. Such creep motion can be detected anywhere in the heap. Although these above studies are useful to the development of the dynamics of granular material, there is still a lack of the systematic study of the description of the surface flows in heaps. The difficulty lies in the coexistence of both liquid and solid behaviours of the material when a layer of granular material flows on the surface of a heap.

In this work, flows of granular material on 'non-frozen' and 'frozen' heaps are investigated numerically and theoretically. The granular flow on a 'non-frozen' heap is first numerically studied using the discrete element method (DEM). The flow profiles of the flow and the creep motion of particles in the heap are investigated. The flow on a 'frozen' static heap is also simulated, and compared with the one on the corresponding 'non-frozen' heap. The continuum model proposed by Jop et al. [9] is then developed to predict theoretically the depth of the surface flow on a static heap. The theoretical results are also compared with the DEM simulation results and the experimental ones in the literature. 


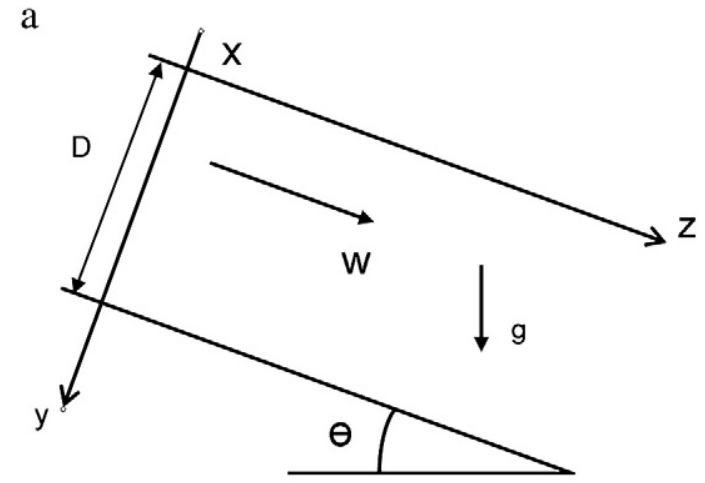

b

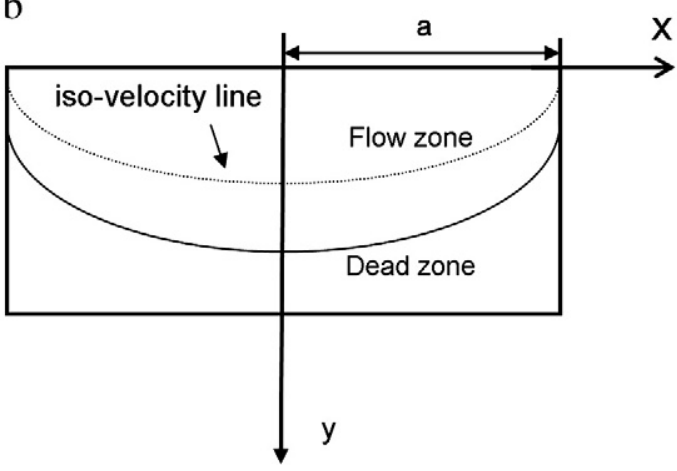

Fig. 2. Sketch of: (a), surface flow on a static heap and the coordinates fixed on it; and (b), iso-velocity lines in a cross-sectional plane for the theoretical study.

from the walls, with the $x$-axis horizontally along the surface, the $y$ axis perpendicular to the surface downwards, and the $z$-axis along the surface downwards.

For a steady-state flow, the velocities of particles are oriented by the $z$-axis and do not depend on $z$. Therefore, we only need to consider the velocity field with a set of iso-velocity lines on a cross-section plane $(x, y)$ (Fig. 2). The flow is assumed to be non-cohesive and satisfy a conical yield criterion and a co-axiality between the stress and strain rate tensor, with no dilatancy $[9,10]$ :

$\boldsymbol{\sigma}=-P \mathbf{I}+\mu(I) P \frac{\dot{\boldsymbol{\gamma}}}{\|\dot{\boldsymbol{\gamma}}\|}$,

where $\sigma$ is the stress tensor, $P=\sigma_{i i} / 3$ the mean pressure, I the unit matrix, $\dot{\boldsymbol{\gamma}}$ the strain rate tensor, and $\|\dot{\boldsymbol{\gamma}}\|=\sqrt{\dot{\gamma}_{i j} \dot{\gamma}_{i j} / 2}$ the second invariant of $\dot{\boldsymbol{\gamma}}$. $I$ is the inertial number, given by $I=\|\dot{\boldsymbol{\gamma}}\| d / \sqrt{P / \rho}$ (where $d$ is the diameter of particles, and $\rho$ the bulk density). $\mu(I)$ is the coefficient of bulk friction, increasing from $\mu_{\mathrm{s}}$ to a finite value $\mu_{\infty}$ when $I$ increases from zero to infinity, and satisfying $\left(\mu-\mu_{\mathrm{s}}\right) \propto I^{m}$, with exponent $m$ being between 0 and 2 when $I \rightarrow 0$. Accelerated flows may occur for cases with slopes greater than $\mu_{\infty}$, while intermittent avalanche flows may be triggered for cases with slopes lower than $\mu_{\mathrm{s}}$. Since only steady-state flows are concerned, we will not consider the two cases.

The force balance along a slope can be integrated, and leads to an exact differential equation along an iso-velocity line in the crosssection plane. Thus, the shape of the velocity field can be given by a set of parametric equations for the iso-velocity lines. Therefore, similar to the derivations of [11] and [12], we can obtain the equations to determine the iso-velocity line passing through point $(0, h)$ :

$\frac{d x}{d s}=\left(\mu_{0}(h)-\tan \theta\right) h+\left(y-y_{\mathrm{w}} A_{\phi}\right) \tan \theta+\mu_{\mathrm{w}}\left(y_{\mathrm{w}}\right) y_{\mathrm{w}} \sqrt{1-\delta^{2}} A_{\phi}$,

$\left(\frac{d y}{d s}\right)^{2}=\mu^{2} y^{2}-\left(\frac{d x}{d s}\right)^{2}$ where $s$ is a curvilinear co-ordinate along the iso-velocity line. $\mu_{0}(h)$ is the friction coefficient at the point $(0, h), \mu_{\mathrm{w}}\left(y_{\mathrm{w}}\right)$ is the friction at the point $\left( \pm a, y_{\mathrm{w}}\right), A_{\phi}=\int_{y}^{h} \frac{d \mu}{d I} I y d y / \int_{y}^{h} \frac{d \mu}{d I} I y d y, \delta=\min \left(\mu_{\mathrm{w}} / \mu_{\mathrm{s}}, 1\right)$.

The boundary between the dead and flow zones can be obtained by considering the limit when the inertial number $I$ tends to zero, i.e. for $\mu \approx \mu_{\mathrm{s}}$ and $A_{\phi} \approx\left(h^{b}-y^{b}\right) /\left(h^{b}-y_{\mathrm{w}}^{b}\right)$, with $b=1-m / 2$, and the depth of a flow layer at the centre, $h_{\max }$, can be given by:

$a=\int_{h_{\max }}^{y_{\mathrm{w}}} \frac{\frac{d x}{d s}}{d s} d y$

Eq. (6) gives an implicit relation between the depth of the flow zone, $h_{\max }$, and the depth of the slip zone at the walls, $y_{\mathrm{w}}$. The latter can be obtained by writing the force balance on the flowing layer contour, that is

$\int_{h_{\max }}^{y_{\mathrm{w}}} \frac{y^{2}}{d y} d y+\delta_{2} \frac{y_{\mathrm{w}}^{2}}{2}-\frac{\tan \theta}{\mu_{\mathrm{s}}} \int_{h_{\max }}^{y_{\mathrm{w}}} \frac{\frac{d x}{d s}}{d s} y d y=0$.

The first and the second terms in Eq. (7) are respectively the scaled basal and lateral shear forces, and the third is the scaled weight along the slope.

The lateral shear force is explicitly known for a smooth wall for which $\delta_{2}=\delta$. For rough walls, the friction on the wall is the bulk friction and its distribution depends on the exact shape of function $\mu(I)$. In such a case, the exact value for $\mu_{\mathrm{w}}$ cannot be obtained but only a range since $\mu(I)$ ranges between $\mu_{\mathrm{s}}$ and $\mu_{\infty}$, and $\delta_{2}$ is a number between 1 and $\mu_{\infty} / \mu_{\mathrm{s}}$.

Eqs. (6) and (7), with Eqs. (4) and (5), form a closed system. Therefore, for the case of a smooth wall (for which the wall friction does not identify to the bulk friction), we can use these equations to determine the ratios $h_{\max } / a$ and $y_{\mathrm{w}} / h_{\max }$ with respect to $R=\tan \theta / \mu_{\mathrm{s}}, \delta$ and the exponent $m$ of the asymptotic behaviour of the bulk coefficient of friction when the shear rate tends to zero. For the case of a rough wall (for which the wall friction identifies to the bulk friction), without the knowledge of the exact form of $\mu(I)$, we only obtain a scope of values using $1<\delta_{2}<\left(\mu_{\infty} / \mu_{\mathrm{s}}\right)$.

\section{Results and discussion}

\subsection{Numerical investigation}

Simple physical and numerical experiments have been first carried out in a 2-D slot model with real front and rear walls. Both experimental and simulation conditions, including the geometrical and operational conditions, are similar. For each experimental run, a triangle shape heap of particles is first formed in the container. Particles are then continuously fed into the hopper, and then discharged to form a shear flow layer on the surface of the heap. All the physical experimental processes have been recorded by a digital video camera. Two cases have been considered for the comparative study: one in which glass beads are used, another where blue and red wooden balls are utilised to form heap and flow layer respectively. It has been observed that the flow patterns for physical and numerical experiments are quite similar in the two cases. Two different layers can be identified: a stable granular heap and a steady-state surface flow upon it. In the experiments with glass beads, the interface between the two layers is not clear in the photos captured in the experiments as all glass beads have the same colour. Therefore, only the results for the case with wooden balls are shown here (Fig. 3). The similarity of the flow patterns in both numerical and physical experiments demonstrates the applicability of the numerical model in this work. Then, in the further numerical experiments, periodic boundary conditions are implemented to eliminate the influence of 
a

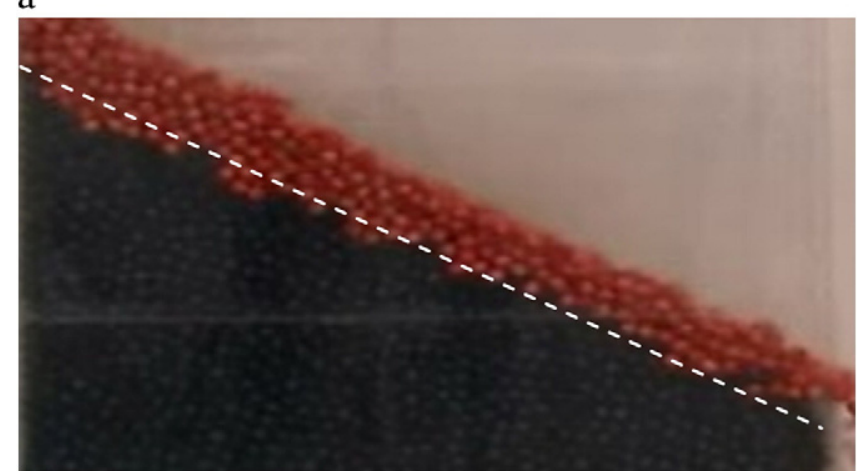

b

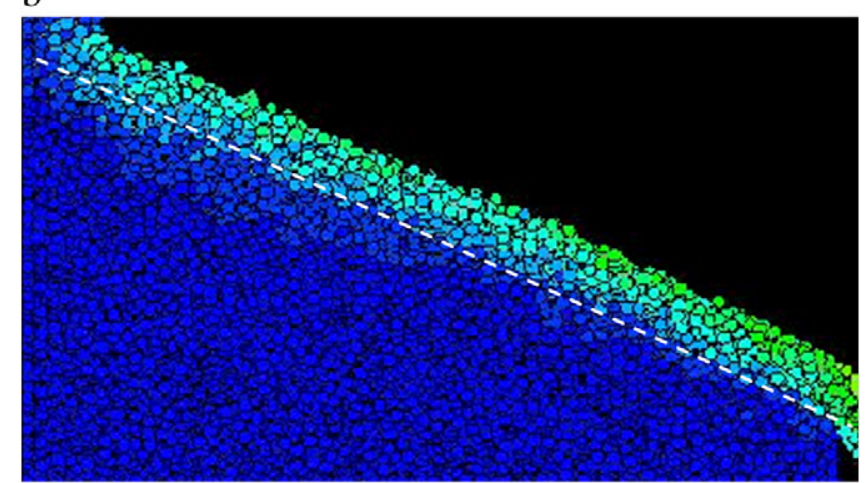

Fig. 3. Flow patterns observed in: (a), physical experiments; and (b), numerica experiments. Most particles above the white line are red wooden balls, while those below the line are mainly blue wooden balls.

the front and rear walls. Glass beads are used to form the heap and surface flow layer.

In the surface flow layer, particle velocities are approximately parallel to the surface of the layer. So only the velocity in the direction parallel to the surface is considered here. For the convenience of description, we employ a coordinates similar to that defined in Fig. 2, which is fixed at the centre of the surface of the flow layer. Fig. 4 shows the variation of the mean velocity along the $y$-axis, where the mean velocity, $v\left(y_{i}\right)$, is the average of the velocities of particles whose mass centres are in the range of $[-l, l] \times\left[y_{i}, y_{i}+\Delta y\right]$. $\Delta y$ and $l$ are one and three blue wooden ball diameters, respectively. It can be observed that the velocity profile contains two distinct parts, respectively corresponding to the two layers. Roughly, the flow layer has a linear velocity distribution, as shown in Fig. 4(b). Increasing y will decrease the velocity. Under the flow layer, the heap undergoes a slow creep motion. Such a motion can be detected anywhere in the heap. Furthermore, our results show that the mean velocity of the particles in the heap exponentially varies with the distance from the surface of the heap. This feature is similar to that obtained by the experiments of Komatsu et al. [13].

It has been known that the flow rate of the particles discharged from a hopper would affect the particle flow on heaps $[18,19]$. This has also been confirmed in the present numerical simulations. The increase of flow rate is realized by increasing the hopper orifice size here. Four sizes, namely $4 \mathrm{~d}, 6 \mathrm{~d}, 8 \mathrm{~d}$ and $10 \mathrm{~d}$ have been considered. It has been shown that the slope and thickness of the flow layer increase with the flow rate. But the slow creep motions of particles in the heap for different flow rates have very similar distributions.

Angular velocity distribution of particles has also been examined in this work. It has been observed that the angular velocities about the $y$ and $z$ axes are very small. The angular velocity about the $x$-axis is relatively large and linearly decreases with $y$ in the flow layer. These results are not shown here for brevity.

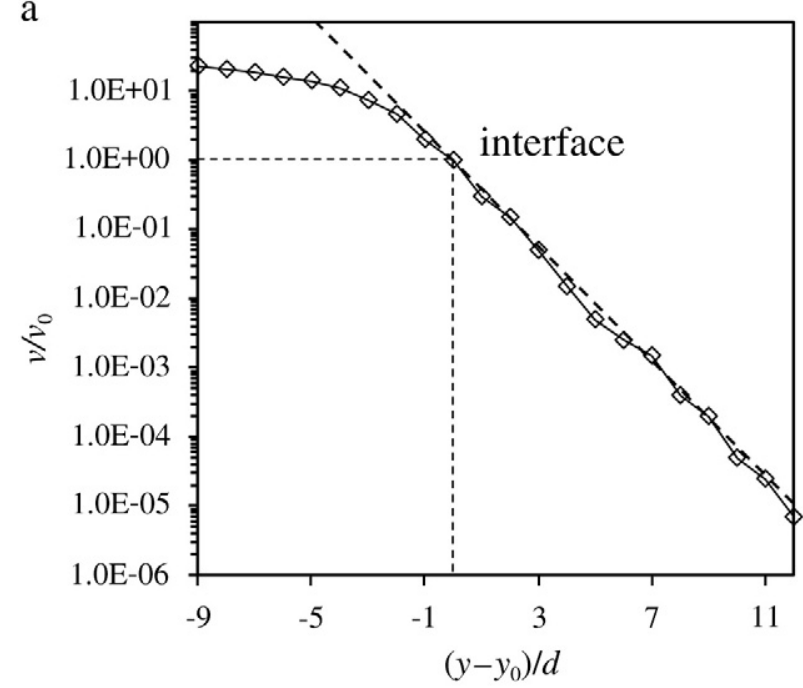

b

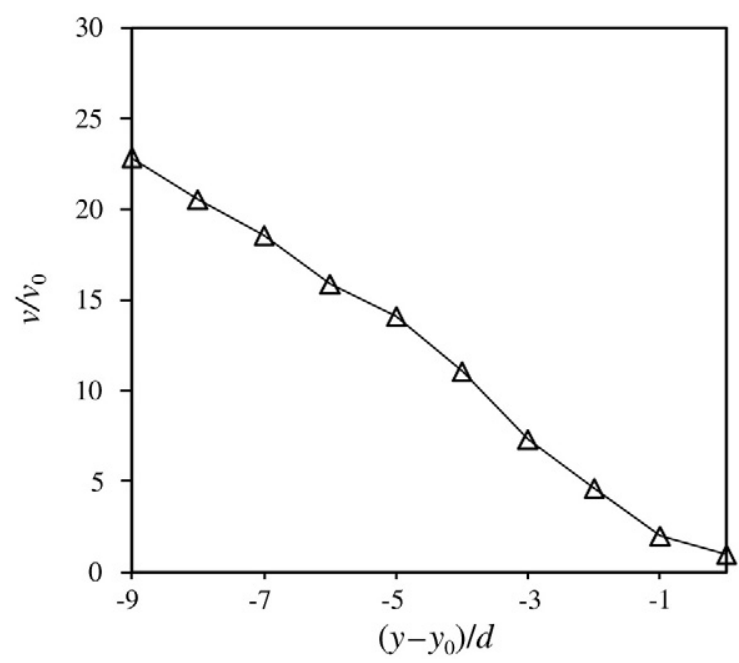

Fig. 4. Mean velocity distribution along the $y$-axis: (a), whole region; and (b), flow layer, where $y_{0}$ is the flow layer thickness, $v$ is the mean velocity of particles, $v_{0}$ is the mean velocity at $y=y_{0}$.

The flow structure of particles is examined in terms of porosity. A snapshot of porosity distribution is shown in Fig. 5(a). It can be seen that the static layer has a lower porosity while the flow layer a higher porosity. The high porosity should be from the relatively unconfined movement of particles. In order to consider the variation of the porosity, four representative regions are chosen: $\mathrm{A}$ is in the flow layer, $B$ the interface region between the static and flow layers, $C$ the lower part near the interface and $D$ the deep region in the heap. As shown in Fig. 5(b), the porosities of the four regions vary with time, although the variation is getting smaller from A to $D$. The large variation in the flow layer means that the particles can move more freely, while the small variation in the deep region indicates that more contacts between particles occur in the static layer. The particles in the regions with lower porosity may move to the regions with higher porosity, which may be the reason causing the creep motion of particles in the heap.

In order to consider the influence of the surface conditions of the heap on the surface flow, a case with the so called 'frozen' static heap is simulated. This case is similar to the surface flow on an inclined plate, a case extensively investigated in the past $[3-5,11,12]$. Fig. 6 shows the velocity profiles along the $y$-axis for both cases with the 'frozen' and 'non-frozen' heaps. The velocity profiles for the two cases are similar, but the value of the velocity for the 'non-frozen' heap is 


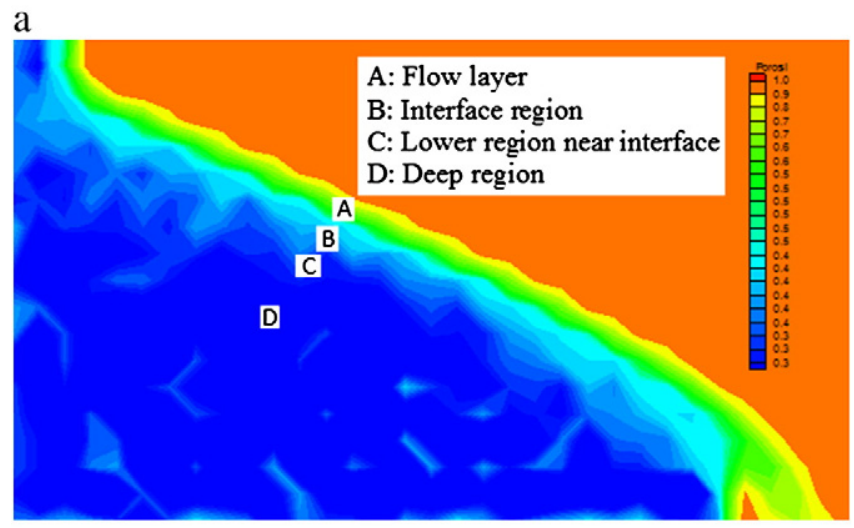

b

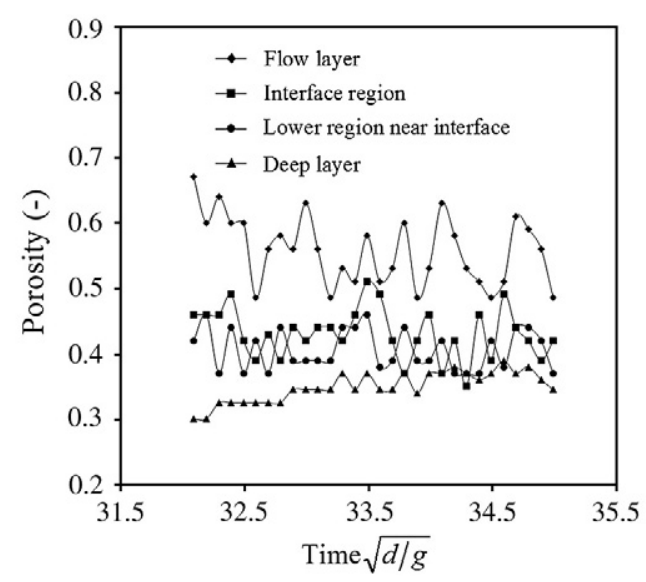

Fig. 5. (a), Porosity distribution in the heap and surface flow layer; and (b), variation of porosity in different regions with time.

larger than that for the 'frozen' heap. The result indicates that the surface conditions of heap affect the velocity profiles of surface flow.

Furthermore, we also have performed DEM simulations to examine the effect of the slope angle of the static heap. Fig. 7 gives the surface velocities and depth velocity profiles at the centre of the channel for slope angles ranging from $22^{\circ}$ to $36^{\circ}$. The flow at $22^{\circ}$ is at the onset of the present model, where the boundary between the flow

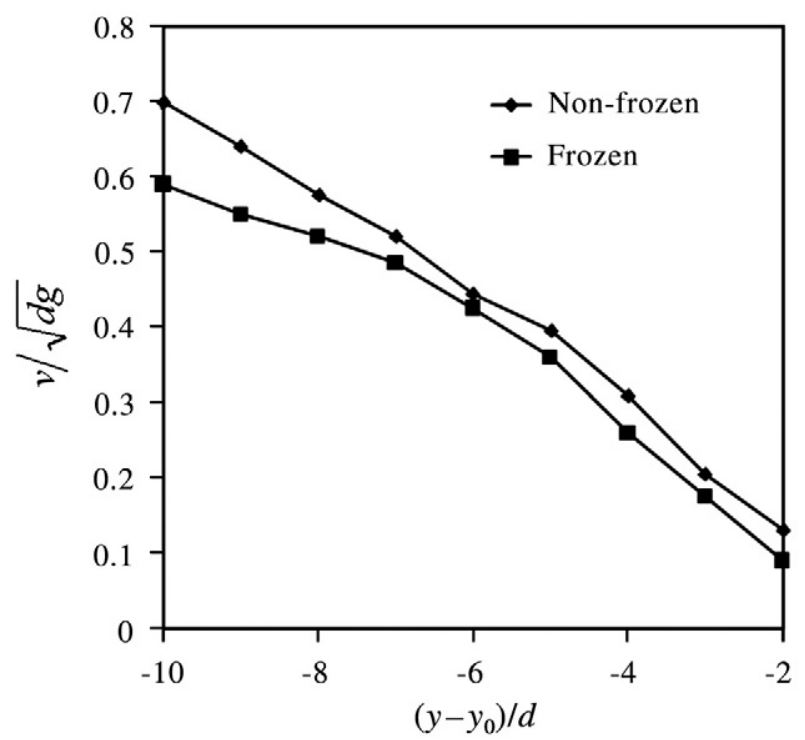

Fig. 6. Velocity profiles of the flow layers for the cases with the 'frozen' and 'non-frozen' heaps.

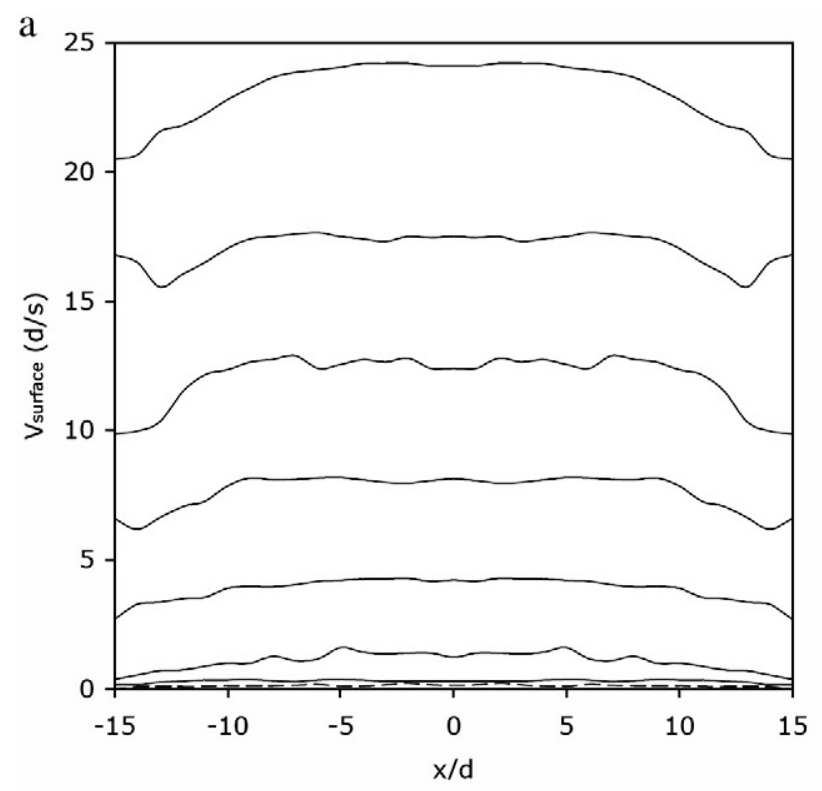

b

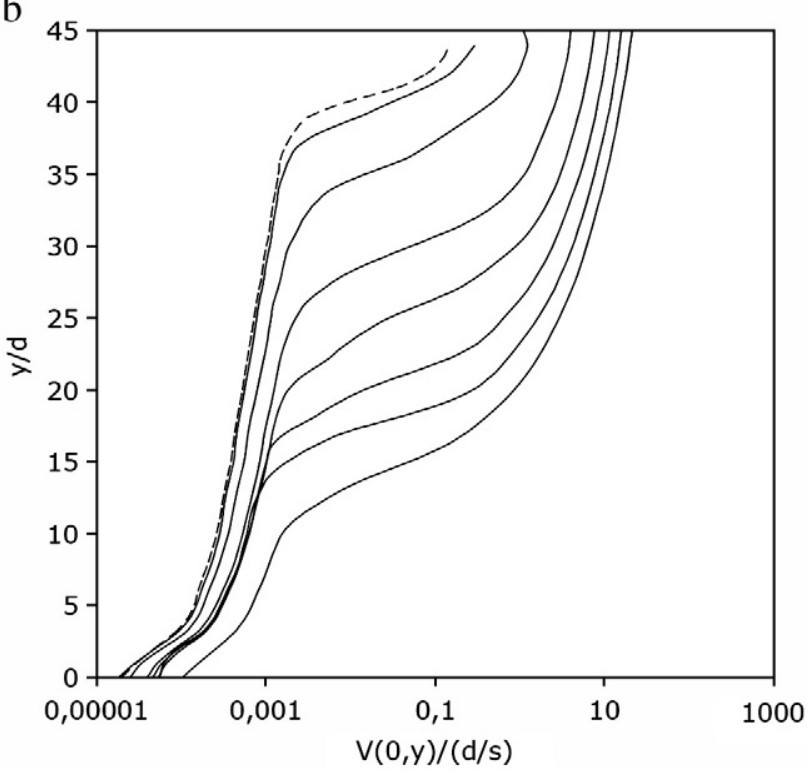

Fig. 7. (a), Surface velocity versus the axial position $x / d$ (from top to bottom, the slope angle $\theta$ ranges from $36^{\circ}$ to $22^{\circ}$ by a $2^{\circ}$ step. Dashed line: $\theta=22^{\circ}$ ); and (b), depth velocity profile for $x=0$ (from bottom to top, the slope angle $\theta$ ranges from $36^{\circ}$ to $22^{\circ}$ by a $2^{\circ}$ step. Dashed line: $\theta=22^{\circ}$ ).

and deadzone does not attain the lateral walls (see the dashed line in Fig. 7(a)). In Fig. 7(b), we notice that the velocities of the particles upon the static heap are non zero, but two distinct zones are observed: a creep zone where the velocity field exponentially decrease with the decrease of the distance from the static heap surface, and an upper zone where the particles have higher velocities. It can be observed that the depth of the flow zone (upper zone) increases with the increase of the slope angle of static heap.

\subsection{Theoretical investigation}

In the theoretical study, the flows on a static heap with smooth or rough lateral walls are considered, and the prediction of the depth of the flow layer is focused on.

Fig. 8 shows the influence of the lateral walls friction conditions on the depth of the flow layer. For smooth lateral walls, the results are represented by the continuous lines. The thick one is for $\mu_{\mathrm{w}}=\mu_{\mathrm{s}}$ and 


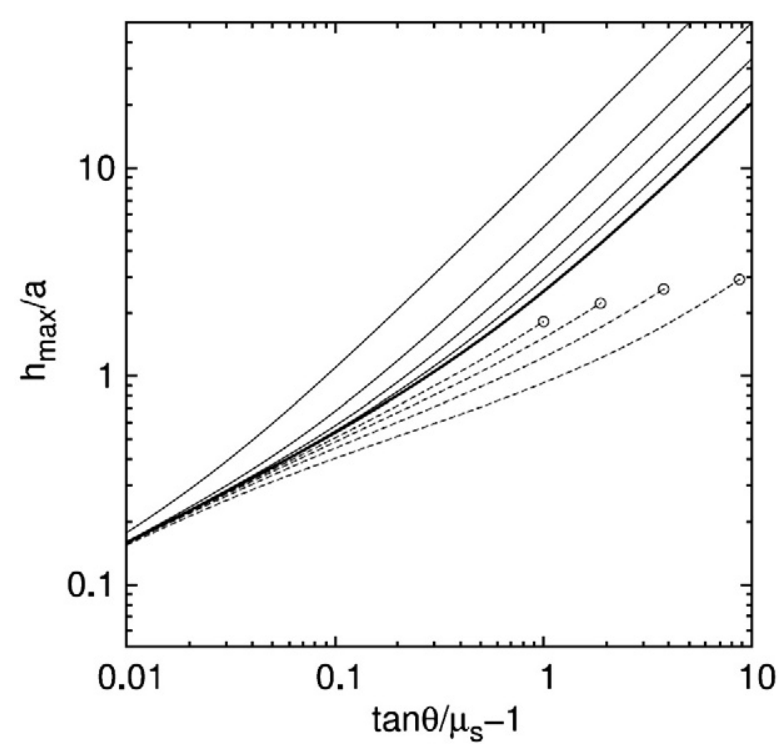

Fig. 8. Depth of the flow layer scaled by the half-width of the heap versus the relative difference between the heap slope and the static coefficient of friction. Continuous lines: smooth wall with $\mu_{\mathrm{w}} / \mu_{\mathrm{s}}=1$ (thick line), $0.8,0.6,0.4$ and 0.2 (thin lines from bottom to top). Dashed lines: minimum value for rough wall with $\mu_{\infty} / \mu_{\mathrm{s}}=2,3,5$ and 10 . These lines end at $R=\mu_{\infty} / \mu_{\mathrm{s}}$ (represented by circles).

the thin ones are for $\delta=0.2,0.4,0.6$ and 0.8 . It can be seen that the depth is affected by the wall friction. The smaller the wall friction, the bigger the depth. The dependence of the depth on parameter $m$ is small, so the results in Fig. 8 remain valid for all the values of $m$ between 0 and 2. For the case of rough walls, we have considered different $\mu_{\infty} / \mu_{\mathrm{s}}$ values, which are represented by the dashed lines below the thick continuous line in Fig. 8. These dashed lines are for $\mu_{\infty} /$ $\mu_{\mathrm{s}}=2,3,5$ and 10. It can be observed that the larger the ratio, the lower the flow layer depth. Note that these dashed curves end at a point. This is because there is no steady-state solution when the heap slope is greater than $\mu_{\infty}$.

Fig. 9 shows the ratio $y_{\mathrm{w}} / h_{\max }$ as a function of the relative difference between the slope and static coefficient of friction for the same values of the properties used in Fig. 8. Similar to the depth of the

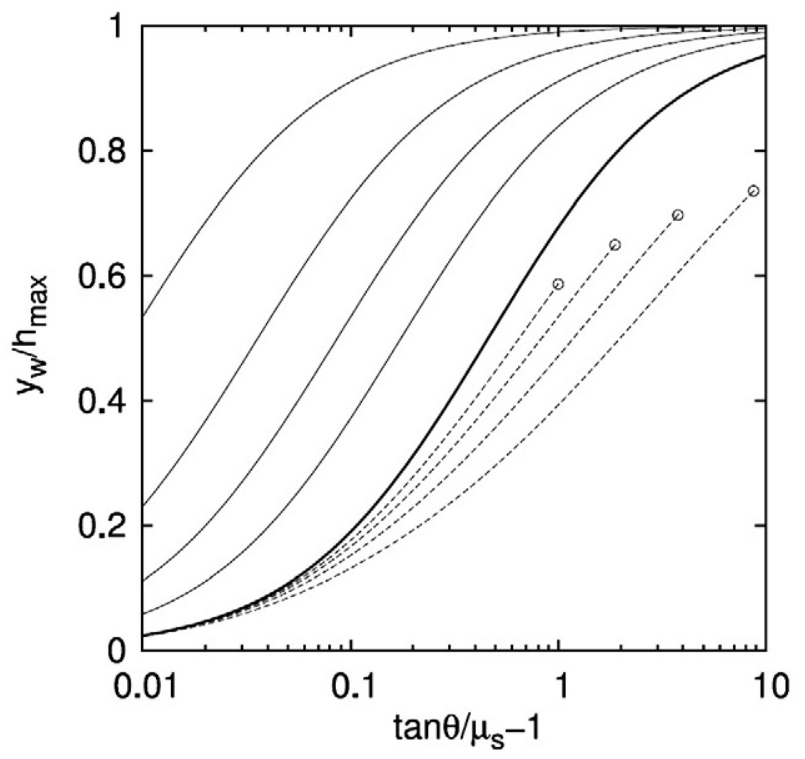

Fig. 9. Depth of the flow layer at the walls scaled by the half-width of the heap versus the relative difference between the slope and the static coefficient of friction. The same symbols as in Fig. 8 have been used. flow at the centre, the depth at walls decreases with the increase of the wall friction for smooth lateral walls or ratio $\mu_{\infty} / \mu_{\mathrm{s}}$ for rough walls.

We can also investigate the asymptotic behaviour when the heap inclination angle tends to the angle of repose $(R \rightarrow 1)$ or when it tends to the value with $R \gg 1$. The asymptotic development of Eqs. (6) and (7) for $R$ close to 1 leads to: $h_{\max } \sim(2 \sqrt{6} / \pi) a \sqrt{R-1} f(m)$, and $y_{\mathrm{w}} / h_{\max } \sim 1-3(R-1)$, where $f(m)$ is a function which ranges from $1.015 \pm 0.001$ to 1 when $m$ ranges from 0 to 2 . Practically, the asymptotic behaviour only depends on the relative difference between the actual slope and the slope at the onset of flow. For high inclination angles, the asymptotic behaviour is: $h_{\max ~} 2 a R / \delta_{2}$, and $y_{\mathrm{w}} / h_{\max } \sim 1-\delta_{2}\left(1-\sqrt{1-\delta^{2}}\right) /(2 R \delta)$, but remains a limit since there is no steady-state solutions for $\tan \theta>\mu_{\infty}$, i.e. $R>\delta_{2}$.

The theoretical results obtained for the depth of a granular surface flow are compared with the numerical results obtained in the above section. From Fig. 7, we can determine the values of the depth of the flow at the centre $h_{\max }$ and the depth of the flow at the lateral walls $y_{\mathrm{w}}$ for different slope angle, as shown in Fig. 10, where $h_{\max }$ and $y_{\mathrm{w}}$, scaled by the half-width of the channel, versus the slope angle are
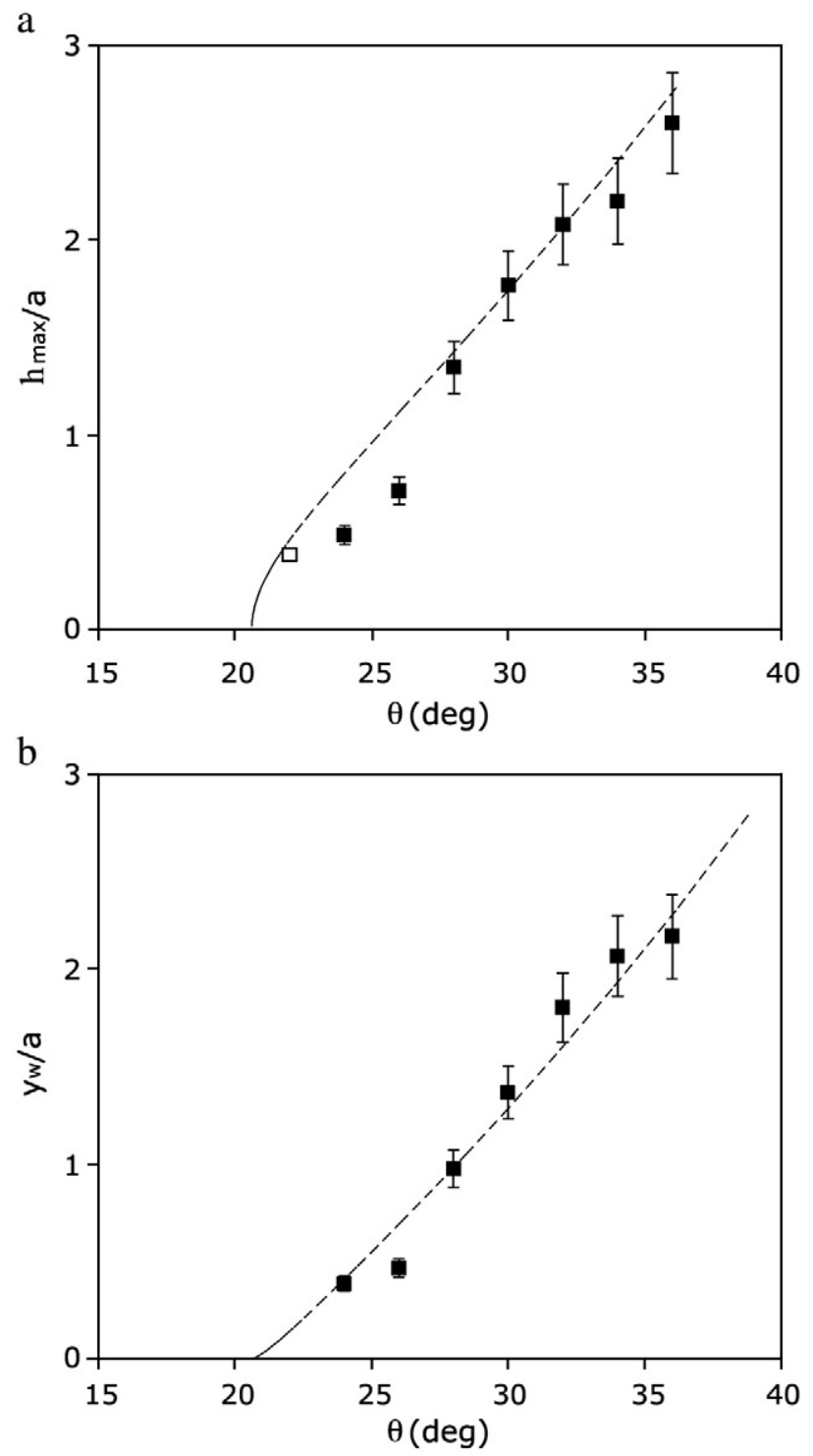

Fig. 10. (a), Depth of the flow layer scaled by the half-width of the heap versus the slope angle $\theta$; and (b), depth of the flow layer at the walls scaled by the half-width of the heap versus the slope angle $\theta$. Data points: results from DEM experiments. The black points are for a slope angle higher than $24^{\circ}$. The white point is for $\theta=22^{\circ}$, for which there is no slip at the walls. Dashed line: theory for $\phi_{\mathrm{s}}=20.6^{\circ}$ and $\mu_{\mathrm{w}} / \mu_{\mathrm{s}}=0.8$. 


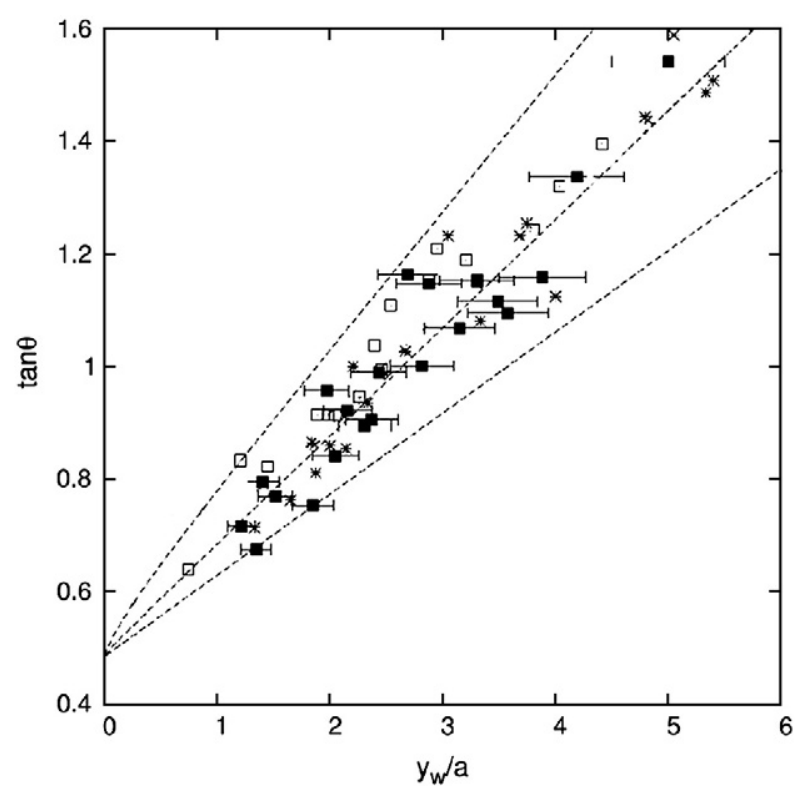

Fig. 11. Heap slope versus the depth at the wall scaled by the half-width of the heap. Stars, black and white squares: experimental results of Taberlet et al. [20] for respectively a channel width of 5,10 and $20 \mathrm{~mm}$. Dashed lines: present theoretical results for a smooth wall (from bottom to top, $\mu_{\mathrm{w}} / \mu_{\mathrm{s}}=0.6,0.8$ and 1 ) and $\mu_{\mathrm{s}}=0.52$.

given. The relevant theoretical results are also given in this figure for comparison. Two variables, the apparent static angle of friction $\phi_{s}=20.6^{0} \pm 1^{0}$ and the ratio $\mu_{\mathrm{w}} / \mu_{\mathrm{s}}=0.8 \pm 0.1$, have been adjusted in order to fit both $h_{\max }$ and $y_{\mathrm{w}}$ with the present theory within a $10 \%$ error.

Taberlet et al. [20] have reported their experimental results on the depth at the walls of granular flows in narrow channels. Here, we attempt to compare our theoretical results with the measured ones. In their experimental study, $y_{\mathrm{w}}$ was not considered. But we can estimate the value of $y_{\mathrm{w}}$ from the velocity profile they presented in [20]. It was found that $y_{\mathrm{w}}$ is approximately 50 to $70 \%$ higher than the depth they obtained based on the extrapolation of the slope at the inflexion point of the velocity profile. The data are reported in Fig. 11 with the corresponding error bars (stars, black and white squares for a channel width of 5, 10 and 20 times the particle size respectively). In this figure, the theoretical prediction obtained based on the rheology proposed by Jop et al. [9] and smooth walls (similar to the conditions of the experiments) is also shown to compare with the experimental data. Since the coefficient of friction between the granular material and the glass windows is not documented in the study of Taberlet et al. [20], we compare the experimental data points with the theoretical results obtained with different wall friction: $\mu_{\mathrm{w}}=\mu_{\mathrm{s}}$ (upper dashed line), $\mu_{\mathrm{w}}=0.8 \mu_{\mathrm{s}}$ (middle dashed line), and $\mu_{\mathrm{w}}=0.6 \mu_{\mathrm{s}}$ (lower dashed line). It can be observed that there is a qualitatively good agreement between the experiments and the theory with a smooth wall whose friction is about $80 \%$ the static bulk friction, and a static bulk friction $\mu_{\mathrm{s}}=\tan \left(27.4^{\circ}\right)$.

The agreements based on the above comparisons confirm the applicability of the theory proposed by Jop et al. [9] to the description of the flow profiles of granular surface flow on static heaps.

\section{Conclusions}

Surface flows on 'non-frozen' and 'frozen' granular heaps have been studied numerically using the discrete element method. It has been observed that the mean velocity of the surface flow on 'nonfrozen' heap exhibits a linear relationship with the distance from the heap surface. Slow creep motion can be observed anywhere in the heap. The mean velocity of the particles in the heap decays exponentially with the distance from the heap surface. The existence of such a creep motion may be attributed to the variation of the porosity distribution of the heap. The granular flow on a 'frozen' static heap is also simulated, and compared with the one on the corresponding 'nonfrozen' heap. The results show that the surface conditions of the heap, to some extent, affect the flow upon it. In particular, the 'non-frozen' heap surface would lead to a larger flow velocity.

The surface flow on static heaps is also examined theoretically using the continuum model recently developed by Jop et al. [9]. The theoretical study illustrates that the depth of the surface flow can be exactly obtained in the case of a smooth wall, and within a range in the case of a rough wall, irrespectively of the detailed knowledge of the relationship between the coefficient of friction and the strain rate. Thus, we can readily predict the depth of flow layer on static heaps. The study of the steady-state surface flow on a heap indicates that the flow layer depth depends on the ratios: wall to bulk static friction $\mu_{\mathrm{w}} /$ $\mu_{s}$, particle size to heap width $d / a$ for a smooth wall. More precisely, the depth decreases with the increase of the wall friction for smooth lateral walls or ratio $\mu_{\infty} / \mu_{\mathrm{s}}$ for rough walls. In addition, the theoretical results have been compared with the DEM simulation results and the experimental ones in literature. Qualitatively good agreements have been observed, which confirms the applicability of the theory proposed by Jop et al. [9] to the description of the flow profiles of granular surface flow on static heaps.

\section{List of symbols}

a half-width of the channel heap ( $\mathrm{m}$ )

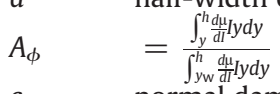

$c_{\mathrm{n}} \quad$ normal damping coefficient between particles

$c_{\mathrm{t}} \quad$ tangential damping coefficient between particles

$d \quad$ diameter of particles $(\mathrm{m})$

$D \quad$ thickness of surface flow layer (m)

E Young's Modulus $\left(\mathrm{kg} / \mathrm{m} \mathrm{s}^{2}\right)$

$\boldsymbol{F} \quad$ inter-particle forces $(\mathrm{N})$

g gravitational acceleration $\left(\mathrm{m} / \mathrm{s}^{2}\right)$

$h_{\text {max }} \quad$ depth of flow layer midway from walls (m)

$I_{i} \quad$ moment of inertia of particle $i\left(\mathrm{~kg} \cdot \mathrm{m}^{2}\right)$

I unit matrix

I inertial number

$\boldsymbol{T}$ torque $(\mathrm{N} \cdot \mathrm{m})$

$m \quad$ mass of particles $(\mathrm{kg})$

$n \quad$ exponent for the asymptotic behaviour of $\mu$ versus $I$ when $I$ tends to zero

$b \quad$ exponent, equals to $1-n / 2$

$P \quad$ mean pressure $(\mathrm{Pa})$

$\mathbf{R} \quad$ vector from the mass centre of particle to contact point ( $\mathrm{m}$ )

$R \quad=\frac{\tan \theta}{\mu_{s}}$

$\left.t \quad \operatorname{time~}^{\frac{1}{\mu_{\mathrm{s}}}} \mathrm{s}\right)$

V velocity $(\mathrm{m} / \mathrm{s})$

$y_{\mathrm{w}} \quad$ depth of slip zone at walls (m)

\section{Greek letters}

$\delta_{t} \quad$ vector of the accumulated tangential displacement between particles $(\mathrm{m})$

$\hat{\boldsymbol{\delta}}_{t} \quad$ unit vector defined by $\hat{\boldsymbol{\delta}}_{t}=\boldsymbol{\delta}_{t} /\left|\boldsymbol{\delta}_{t}\right|$

$\delta_{\mathrm{n}} \quad$ normal displacement between particles $(\mathrm{m})$

$\delta \quad=\min \left(\frac{\mu_{\mathrm{w}}}{\mu_{\mathrm{s}}}, 1\right)$

$v \quad$ Poisson ratio

$\omega$ particle angular velocity, $\mathrm{s}^{-1}$

$\hat{\boldsymbol{\omega}} \quad$ unit vector defined by $\hat{\boldsymbol{\omega}}=\omega /|\omega|$

$\rho \quad$ bulk density $\left(\mathrm{kg} \mathrm{m}^{-3}\right)$

$\boldsymbol{\sigma} \quad$ stress tensor $(\mathrm{Pa})$

$\dot{\gamma} \quad$ strain rate tensor $\left(\mathrm{s}^{-1}\right)$

$\mu \quad$ coefficient of bulk friction 
$\mu_{\mathrm{s}} \quad$ static friction coefficient (with no shear rate),

$\mu_{\infty} \quad$ friction coefficient when shear rate tends to infinity

$\mu_{0}(h) \quad$ friction coefficient at point $(0, h)$

$\mu_{\mathrm{w}}\left(y_{\mathrm{w}}\right) \quad$ friction coefficient at point $\left( \pm a, y_{\mathrm{w}}\right)$

$\mu_{\mathrm{p}} \quad$ friction coefficient between particles

$\delta_{2} \quad$ equals to $\delta$ (smooth wall) or a number between 1 and $\mu_{\infty} / \mu_{\mathrm{s}}$ (rough wall)

$\delta_{t, \max } \quad=\mu_{\mathrm{p}} \frac{2-v}{2(1-v)} \delta_{\mathrm{n}}$

$\theta \quad$ angle of heap surface from the horizontal plane

\section{Subscripts}

$i \quad$ particle $i$

ij $\quad$ between particles $i$ and $j$

$j \quad$ particle $j$

n normal component

$r \quad$ rolling friction

t tangential component

\section{Acknowledgments}

The authors are grateful to the Australian Research Council (ARC) for the financial support of their work. AdR would also like to thank UNSW for hosting his sabbatical leave in 2007.

\section{References}

[1] J. Rajchenbach, Granular flows, Adv. Phys. 49 (2000) 229-256.

2] O. Hungr, A model for the runout analysis of rapid flow slides, debris flows, and avalanches, Can. Geotech. J. 32 (1995) 610-623.
[3] M.A. Kern, F. Tiefenbacher, J.N. McElwaine, The rheology of snow in large chute flows, Cold Reg. Sci. Technol. 39 (2004) 181-192.

[4] S.B. Savage, K. Hutter, The motion of a finite mass of granular material down a rough incline, J. Fluid Mech. 199 (1989) 177-215.

[5] G.D.R. Midi, On dense granular flows, Eur. Phys. J. E. 14 (2004) 341-365.

[6] R.A. Bagnold, Experiments on a gravity free dispersion of large solid spheres in a Newtonian fluid under shear, Proc. R. Soc. Lond. A 255 (1954) 49-63.

[7] O. Pouliquen, N. Renaut, Onset of granular flows on an inclined rough surface: dilatancy effects, J. Phys. II France 6 (1996) 923-935.

[8] R. Brewster, G.S. Crest, J.W. Landry, A.J. Levine, Plug flow and the breakdown of bagnold scaling in cohesive granular flows, Phys. Rev. E 72 (2005) 061301.

[9] P. Jop, Y. Forterre, O. Pouliquen, A constitutive law for dense granular flows, Nature 441 (2006) 727-730.

[10] A.W. Jenike, A theory of flow of particulate solids in converging and diverging channels based on a conical yield function, Powder Technol. 50 (1987) 229-236.

[11] A. de Ryck, R. Ansart, J.A. Dodds, Granular flows down inclined channels with a strain-rate dependent friction coefficient, part I: non-cohesive materials, Granular Matter 10 (2008) 353-360.

12] A. de Ryck, Granular flows down inclined channels with a strain-rate dependent friction coefficient, part II: cohesive materials, Granular Matter 10 (2008) 361-367.

[13] T.S. Komatsu, S. Inagaki, N. Nakagawa, Creep motion in a granular pile exihibiting steady surface flow, Phys. Rev. Lett. 86 (2001) 1757-1760.

[14] P. Richard, A. Valance, J-F. Metayer, P. Sanchez, J. Crassous, M. Louge, R. Delannay, Rheology of confined granular flows: scale invariance, glass transition, and friction weakening, Phys. Rev. Lett. 101 (2008) 248002.

[15] H.P. Zhu, A.B. Yu, The effects of wall and rolling resistance on the couple stress of granular materials in vertical flow, Phys. A 325 (2003) 347-360.

[16] H.P. Zhu, A.B. Yu, Micromechanic analysis of hopper flow of granular materials, J. Eng. Math. 52 (2005) 307-320.

[17] S.M. Wu, H.P. Zhu, A.B. Yu, P. Zulli, Numerical investigation of crater phenomena in a particle stream impact onto a granular bed, Granular Matter 9 (2007) 7-17.

[18] P.A. Lemieux, D.J. Durian, From avalanches to fluid flow: a continuous picture of grain dynamics down a heap, Phys. Rev. Lett. 85 (2000) 4273-4276.

[19] D.V. Khakhar, A.V. Orpe, P. Andresen, J.M. Ottino, Surface flow of granular materials: model and experiments in heap formation, J. Fluid Mech. 441 (2001) 255-264.

[20] N. Taberlet, P. Richard, A. Valance, W. Losert, Superstable granular heap in a thin channel, Phys. Rev. Lett. 91 (2003) 264301. 